\title{
Alergia e intolerancia a antiinflamatorios no esteroideos en pediatría
}

\author{
Allergy and intolerance to nonsteroidal anti-inflammatory drugs \\ in children
}

Dra. María P. Sarraquigne, Dra. Andrea I. Mariño, Dr. Ricardo Saranz,

Dr. Mauricio Colella, Dra. Karina López, Dra. María del P. Bovina Martijena,

Dr. Claudio Parisi ${ }^{a}$ Dr. Julio Orellana, Dr. Claudio Agüero, Dra. Viviana Seisdedos,

Dr. Alejandro Lozano, Dr. Jorge F. Máspero, Dr. Raúl V. Boudet,

Dr. Juan M. Suárez García, Dra. Gloria Bandin, Dra. Marcela García,

Dra. María E. Gervasoni, Dra. Natalia Luconi, Dra. Mónica Matta Ruffolo,

Dr. Víctor Skrie, Dr. Martín Bózzola, Dr. Norberto Procopio,

Dr. Antonio Sánchez Segovia, Dra. Irene Araoz, Dra. María C. Cassaniti y

Dra. Elsa Mindel, por el Comité Nacional de Alergia.

Colaboradores: Dr. Roque G. Arnolt, Dr. Adolfo Blanco, Dra. María E. Cáceres, Dr. Aldo Cavallo, Dra. Renée Claeys, Dra. Nilda Daguerre, Dra. Patricia Dayán, Dra. Mariana Díaz Gálvez, Dr. Jorge García, Dr. Héctor H. González, Dr. Dante Maldonado, Dra. Betina Menéndez Porfilio, Dr. Fabio Orellano, Dra. Raquel Rodríguez y Dra. Laura Sacia.

\section{RESUMEN}

Los antiinflamatorios no esteroideos son ampliamente recetados en niños. Constituyen la segunda causa de reacciones a medicamentos en pediatría después de los antibióticos betalactámicos; sin embargo, solo una parte de estas son reacciones de hipersensibilidad. La prevalencia de dichas reacciones a antiinflamatorios no esteroideos en niños es del 0,3\% y aumenta al $5 \%$ en asmáticos.

Los mecanismos fisiopatológicos involucrados (inhibición delaciclooxigenasa, hipersensibilidad mediada por inmunoglobulina $\mathrm{E}$, linfocitos $\mathrm{T}$ reactivos y / o afectación de la inmunidad innata) darán lugar a diferentes entidades clínicas con sintomatología dispar.

La confusión con síntomas propios de procesos virales y la variabilidad clínica hacen del diagnóstico de certeza un verdadero desafío. Una historia clínica detallada, análisis de laboratorio, pruebas cutáneas y de provocación controlada permitirán definir estrategias para cada paciente en particular sinetiquetar como alérgico a un niño que no lo es ni exponer a riesgos innecesarios a quien está sensibilizado.

Filiación de los autores ver página 9.

\section{Correspondencia:}

Dra. María P.

Sarraquigne:

paula sarra@hotmail.com

Financiamiento:

Ninguno.

Conflicto de intereses:

Ninguno que declarar.

Recibido: 11-7-2019

Aceptado: 29-7-2019
Palabras clave: alergia, pediatría, antiinflamatorios no esteroideos, hipersensibilidad.

\footnotetext{
ABSTRACT

Nonsteroidal anti-inflammatory drugs are widely prescribed in children. They are the second cause of drug's reactions in pediatrics after beta-lactam antibiotics, however only a part of them are hypersensitivity reactions. The prevalence of these reactions to nonsteroidal anti-inflammatory drugs in children is $0.3 \%$, increasing to $5 \%$ in asthmatics.

The different physiopathological mechanisms involved (inhibition of cyclooxygenase, immunoglobulin E-mediated hypersensitivity,
}

reactive $\mathrm{T}$ lymphocytes and/or disturbance of innate immunity) will cause different clinical entities with diverse symptoms.

The confusion between the common symptoms of a viral infection and a hypersensitivity reaction, and the variability of the clinical presentations make diagnosis a real challenge.

A detailed clinical history, laboratory, skin and controlled provocation tests will provide strategies for each patient, without labeling a child who is not an allergic one, or taking unnecessary risks with those who are sensitized. Key words: allergy, pediatrics, non-steroidal antiinflammatory agents, hypersensitivity.

http: / / dx.doi.org/10.5546/ aap.2020.S1

Cómo citar: Sarraquigne MP, Mariño AI, Saranz R Colella M, et al. Alergia e intolerancia a antiinflamatorios no esteroideos en pediatría. Arch Argent Pediatr 2020;118(1): S1-S11.

\section{GLOSARIO}

- AINE: antiinflamatorios no esteroideos.

- RAM: reacción adversa a medicamento.

- RHS: reacción de hipersensibilidad.

- COX: ciclooxigenasa.

- LT: linfocito T.

- IL: interleuquina.

- TSLP: linfopoyetina estromática tímica.

- SNIUAA: urticaria o angioedema y / o anafilaxia inducida por un 
solo antiinflamatorio no esteroideo (del inglés selective non-steroidal antiinflammatory drugs induced urticaria/angioedema or anaphylaxis).

- SNIDR: reacción selectiva retardada inducida por antiinflamatorio no esteroideo (del inglés selective non-steroidal antiinflammatory drugs induced delayed reactions).

- NERD: enfermedad respiratoria exacerbada por antiinflamatorios no esteroideos (del inglés non-steroidal antiinflammatory drugs exacerbated respiratory disease).

- ASA: asma, rinitis/poliposis nasal e intolerancia a la aspirina.

- NECD: enfermedad cutánea exacerbada por antiinflamatorios no esteroideos (del inglés non-steroidal antiinflamatory drugs exacerbated cutaneous disease).

- UCE: urticaria crónica espontánea.

- NIUA: urticaria o angioedema inducidos por antiinflamatorios no esteroideos (del inglés non-steroidal antiinflammatory drugs induced urticaria/angioedema).

- NET/SSJ: necrólisis epidérmica tóxica/ síndrome de Stevens-Johnson.

- AAS: ácido acetil salicílico.

- DRESS: reacción a drogas con eosinofilia y síntomas sistémicos (del inglés drug rash with eosinophilia and systemic symptoms).

- PEGA: pustulosis exantemática generalizada aguda.

- PPC: prueba de provocación controlada.

\section{INTRODUCCIÓN}

Este documento tiene como objetivo relevar información actualizada sobre las reacciones alérgicas a los antiinflamatorios no esteroideos (AINE) y brindarle al pediatra herramientas diagnósticas y terapéuticas de abordaje integral para evitar riesgos y mejorar la calidad de vida de los niños.

Las reacciones adversas a medicamentos (RAM) se definen como cualquier efecto perjudicial o no deseado que ocurre tras la administración de una dosis medicamentosa normalmente utilizada en la especie humana para la profilaxis, el diagnóstico y/o el tratamiento de la enfermedad o para la modificación de una función biológica. ${ }^{1}$

Se habla de reacción de hipersensibilidad (RHS) cuando la RAM es producida por un mecanismo inmunológico. Las RHS se caracterizan por su especificidad y reproducción con la reexposición al fármaco. ${ }^{2}$ Los mecanismos fisiopatológicos involucrados responden a la clasificación de Gell y Coombs ${ }^{3}$ (Figura 1).

\section{EPIDEMIOLOGÍA}

Los AINE son fármacos con actividad antipirética, analgésica, antiinflamatoria y, en distinto grado, inhibitoria de la función plaquetaria. Tienen un amplio uso a nivel mundial, muchas veces, sin prescripción médica, estimado en 80 dosis por persona al año. Esto

FIgURA 1. Tipos de reacciones de hipersensibilidad

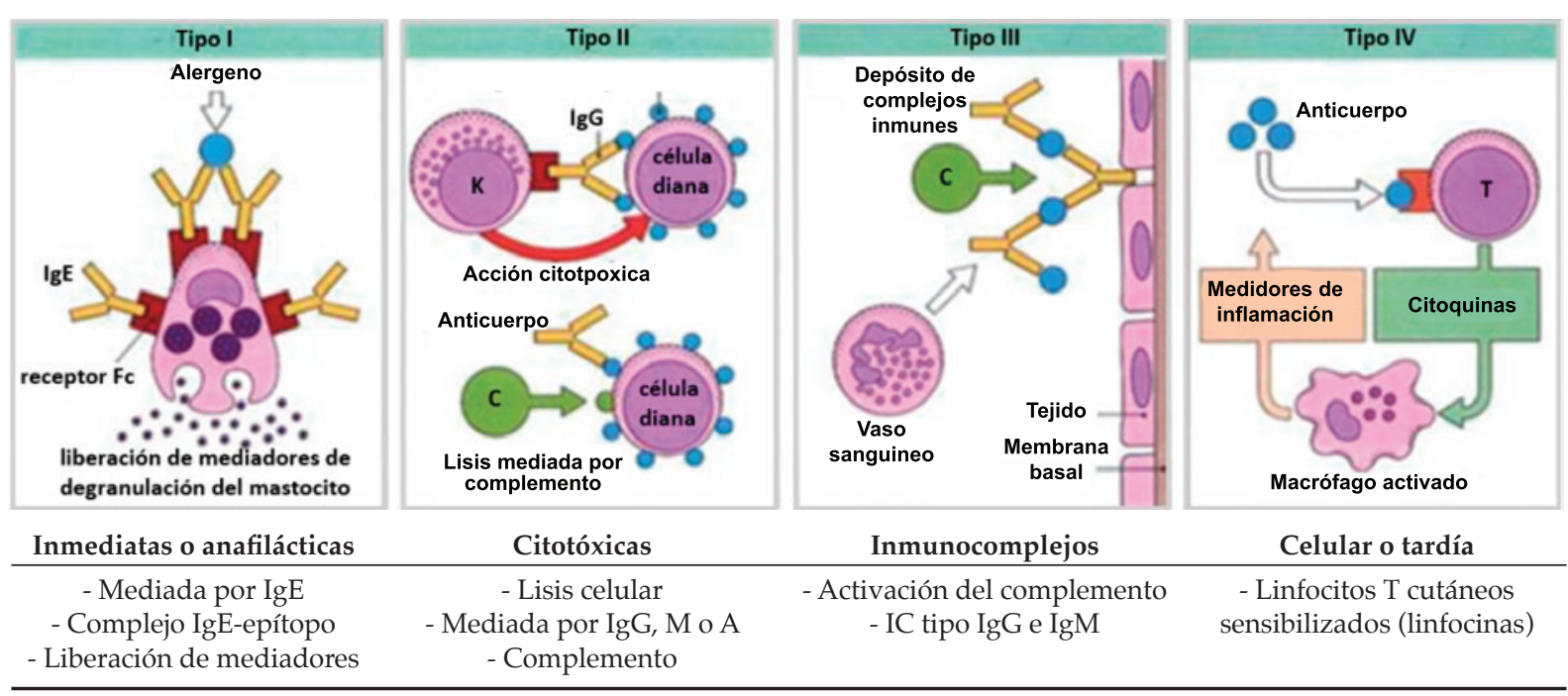

Tomado y adaptado de Brugaletta Matheus D. Hospital Universitario Virgen de la Arrixaca, Servicio de Alergología, 2009. Fc: fracción constante; IgG: inmunoglobulina G; IgE: inmunoglobulina E; IgM: inmunoglobulina M. 
favorece que sean una de las principales causas de RAM. ${ }^{4,5}$

Son los medicamentos más recetados en los niños durante una infección viral. ${ }^{4,6}$ Se pueden confundir síntomas propios de estas enfermedades con una RHS y etiquetar como alérgico al niño sin haber realizado ninguna evaluación alergológica. Solo una minoría de estas reacciones se confirma como RHS. ${ }^{4}$

Constituyen la segunda causa de reacciones a medicamentos en pediatría después de los antibióticos betalactámicos. La prevalencia de RHS a AINE en la población general se estima del $0,6 \%$ al $5,7 \%$, mientras que, en los niños, es de alrededor del 0,3 \% $\%^{4-10}$ y aumenta al $5 \%$ en los niños con asma. ${ }^{10}$ En los adultos, puede afectar hasta un $11 \%$ de los pacientes con asma, un 20$60 \%$ de los pacientes con urticaria crónica y hasta el $70 \%$ de los pacientes con poliposis nasal. La prevalencia de anafilaxia es del 0,01 \% ${ }^{11}$ (Tabla 1). ${ }^{12}$

Las reacciones cruzadas a distintos AINE pueden ocurrir en el $75 \%$ de todos los pacientes con reacciones cutáneas agudas a estas drogas. ${ }^{13}$ Las RHS a AINE se describen más frecuentemente asociadas a pirazolonas, ibuprofeno, diclofenac, aspirina y, en menor medida, paracetamol. ${ }^{13}$ Este último ha sido el medicamento de elección en los pacientes con intolerancia a los AINE. Su rango de reacciones adversas varía entre el 6,7 \% y el $40 \%$, dependiendo de la dosis utilizada, ya que algunos pacientes toleran dosis bajas, pero no su incremento. ${ }^{5}$

No se han publicado grandes estudios poblacionales en los pacientes menores de
14 años, y los únicos hallazgos disponibles provienen de informes de casos aislados y series pequeñas. ${ }^{14,15}$

\section{FISIOPATOGENIA}

La clasificación de los AINE se puede realizar según la estructura química o por su efecto sobre la ciclooxigenasa 1 y 2 (COX-1 y COX-2). La Tabla 2 los clasifica según su efecto sobre dicha enzima y permite distinguir grupos de AINE con potenciales reacciones cruzadas, lo que tiene implicancias clínicas y terapéuticas. ${ }^{16,17}$

Las RHS a AINE se producen por diferentes mecanismos patogénicos. Las más frecuentes son provocadas por un mecanismo no inmunológico que inhibe la ciclooxigenasa y altera el metabolismo del ácido araquidónico de la membrana celular. ${ }^{17}$ En algunas ocasiones, predomina un mecanismo inmunológico de hipersensibilidad mediada por inmunoglobulina E (IgE), linfocitos T (LT) reactivos y/o afectación de la inmunidad innata. ${ }^{18,19}$

El mecanismo no inmunológico se corresponde con AINE que producen inhibición de la COX-1, lo que se asocia a un incremento de la producción de leucotrienos desde mastocitos, basófilos y eosinófilos, y una disminución de la producción de prostaglandinas inhibitorias, principalmente, prostaglandina E2 (PGE2), y de la actividad de su receptor ${ }^{17,18}$ (Figura 2). Esto genera contracción del músculo liso bronquial, vasodilatación y edema, con las consecuentes manifestaciones clínicas respiratorias, cutáneas y / o sistémicas. Los síntomas se presentan ante la ingesta de AINE

TABLA 1. Prevalencia de sensibilización a ácido acetil salicilico según la enfermedad de base ${ }^{12}$

\begin{tabular}{|c|c|c|}
\hline \multirow[t]{2}{*}{ Asma } & Niños & $\begin{array}{l}\text { Menores de } 10 \text { años: infrecuente } \\
\text { Mayores de } 10 \text { y jóvenes < de } 20 \text { años: } \\
\text { el } 10 \%\end{array}$ \\
\hline & Adultos & $\begin{array}{l}\text { Solo asma: el } 10-20 \% \\
\text { Asma y rinosinusitis: el 30-40 \% } \\
\text { Historia sugestiva de intolerancia a } \\
\text { AAS: el } 60-85 \%\end{array}$ \\
\hline Urticaria crónica & $\begin{array}{l}\text { Enfermedad controlada: el 20-30\% } \\
\text { Enfermedad inestable: el 50-80\% }\end{array}$ & \\
\hline Urticaria aguda & $\begin{array}{l}\text { Por AAS: el 1-2 } \% \\
\text { Por AINE en general: el 1-3 } \%\end{array}$ & \\
\hline Anafilaxia & Menor del $1 \%$ & \\
\hline Meningitis aséptica & Infrecuente & \\
\hline Neumonitis por hipersensibilidad & Infrecuente & \\
\hline
\end{tabular}

AAS: ácido acetil salicílico; AINE: antiinflamatorios no esteroideos. 
que comparten la propiedad de inhibir la COX-1, aunque no estén químicamente relacionados, por lo que estos pacientes reaccionan ante más de una droga. El paracetamol presenta algunas particularidades. Es un inhibidor débil, in vitro, tanto de la COX-1 como de la COX-2, que requiere altas dosis para provocar esta inhibición. ${ }^{20}$ Posiblemente, inhiba otra isoforma de COX-2, la COX-3, que sería producto del mismo gen que codifica la COX-2, aunque con características moleculares diferentes. ${ }^{21}$ Esto explicaría que algunos pacientes tuvieran reacciones con dosis altas de paracetamol, pero toleraran las bajas.
El mecanismo inmunológico específico de la droga mediado por IgE es menos frecuente. En este caso, las reacciones se producen por la administración de un solo fármaco o grupo de fármacos.

Recientemente, se ha reportado un defecto de la respuesta inmune innata, a través de células linfoides innatas tipo 2 (ILC-2) con sobreexpresión de interleuquina 33 (IL-33) y linfopoyetina estromática tímica (thymic stromal lymphopoietin; TSLP, por sus siglas en inglés). Estas alarminas producidas por el epitelio pueden desencadenar activación de mastocitos e inflamación eosinofilica ${ }^{19}$ (Figura 2).

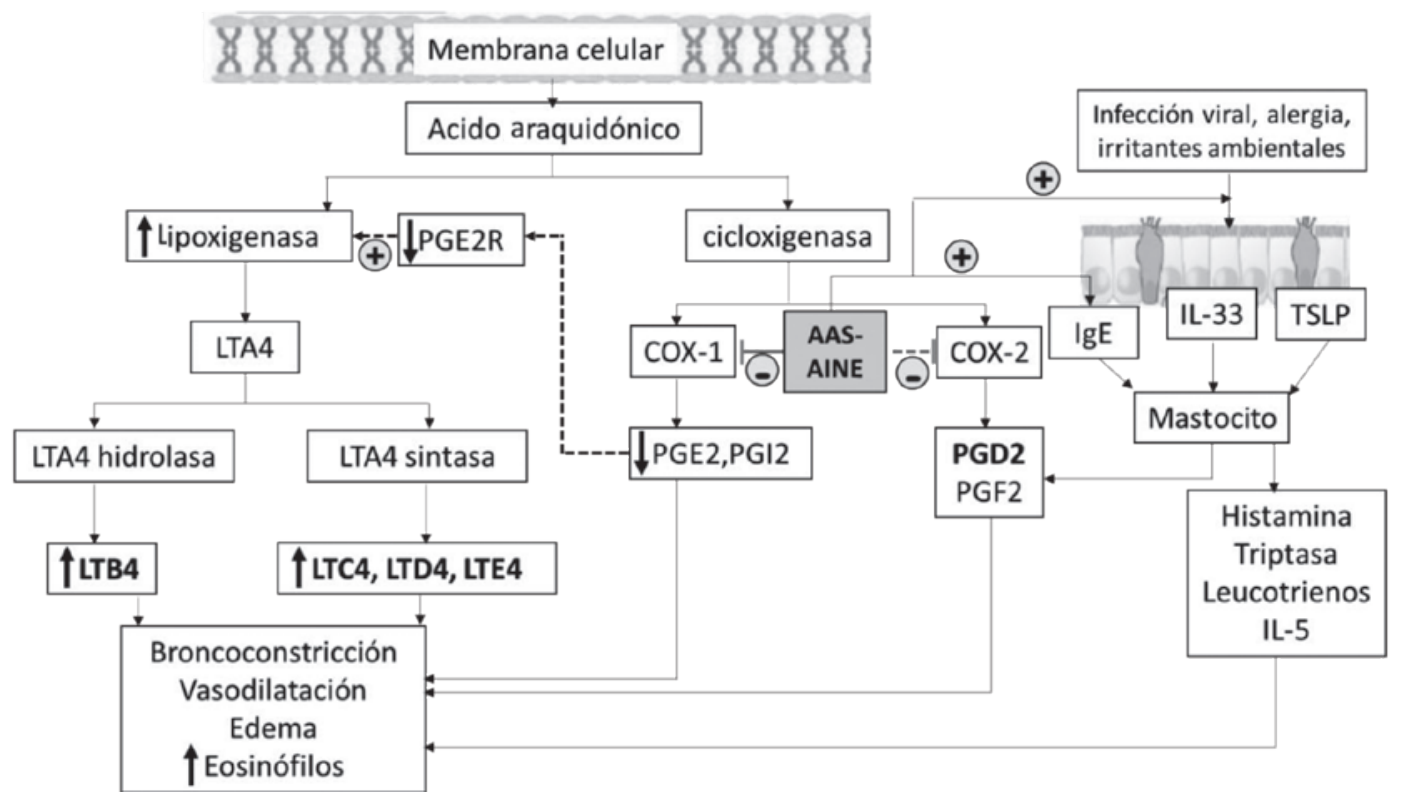

Fuente: Elaboración propia.

LT: leucotrieno; PG: prostaglandina; COX-1: ciclooxigenasa-1; COX-2: ciclooxigenasa-2; PGE2R: receptor de prostaglandina E2; TSLP: linfopoyetina estromática tímica; AAS: ácido acetil salicílico; AINE: antiinflamatorios no esteroideos; IgE: inmunoglobulina E; IL: interleuquina.

TABla 2. Clasificación de antiinflamatorios no esteroideos según su efecto sobre la ciclooxigenasa 1 y 2 (adaptado de Modena $B$, White A, Woessner K. Aspirin and nonsteroidal antiinflammatory drugs hypersensitivity and management. Immunol Allergy Clin North Am. 2017)

Efecto sobre ciclooxigenasas

Máximo efecto inhibitorio sobre

COX-1 y COX-2 con poca selectividad

Efecto inhibitorio preferencial sobre COX-2 y parcial sobre COX-1

\section{Fármacos}

Ácido acetil salicílico, * piroxicam, ${ }^{*}$ sulindac, ibuprofeno,

${ }^{*}$ ketorolac, ${ }^{*}$ naproxeno, ${ }^{*}$ ketoprofeno, ${ }^{*}$ indometacina,

* diclofenac*

Etodolac, meloxicam, ${ }^{*}$ nimesulida, nabumetone.

Gran efecto inhibidor sobre COX-2 y mínima acción inhibitoria sobre COX-1

Rofecoxib, etoricoxib, * valdecoxib, lumiracoxib, celecoxib*

Débil efecto inhibidor sobre COX-1 y COX-2

Paracetamol, * diflunisal, sulfasalazina*

* Disponibles en vademécum de la Argentina.

COX-1: ciclooxigenasa-1; COX-2: ciclooxigenasa-2. 


\section{MANIFESTACIONES CLÍNICAS}

Existen diferentes entidades clínicas de hipersensibilidad a AINE. Para caracterizarlas, se sugiere una clasificación basada en el mecanismo fisiopatológico subyacente, que será distinto si se producen por la ingesta de un solo AINE (reacciones selectivas) o si involucran más de un grupo químico (reacciones por intolerancia cruzada). Estas últimas son las más prevalentes, y el angioedema facial es la presentación clínica más frecuente en los niños. ${ }^{22}$

\section{METODOLOGÍA DIAGNÓSTICA Historia clínica}

Una historia clínica detallada permitirá sospechar el mecanismo patogénico involucrado y definirá el protocolo de estudio para seguir. ${ }^{23}$ Debe incluir el fármaco implicado, la dosis y la vía de administración, las manifestaciones clínicas que llevaron a su uso, los síntomas previos y ulteriores a la toma, el tiempo transcurrido hasta el inicio de los síntomas y los medicamentos concomitantes (Tablas 3 y 4). ${ }^{24,25}$

Se debe interrogar sobre trastornos crónicos subyacentes, como asma bronquial, rinosinusitis crónica, pólipos nasales, urticaria y/o angioedema crónicos. ${ }^{13}$ Si bien la historia clínica no predice con exactitud la aparición de reacciones futuras, los episodios que involucren síntomas respiratorios y/o anafilaxia tienen un valor predictivo mayor que la presencia de síntomas cutáneos aislados. ${ }^{13}$

TABLA 3. Manifestaciones clinicas en las reacciones de hipersensibilidad selectivas ${ }^{15,23,26}$

- Mecanismo inmunológico o alérgico

Entidades clínicas

Puntos claves

SNIUAA

- IgE mediado.

- Síntomas dentro de la primera hora de la ingesta.

Urticaria-angioedema y / o anafilaxia después

de la ingesta de un solo AINE estrechamente relacionado con los del mismo grupo. Toleran alternativos no relacionados químicamente.

Independiente de la fuerza de la inhibición de la COX-1. Ibuprofeno, paracetamol o dipirona.

SNIDR

- Mediados por LT.

- Síntomas dentro de

Exantema

las 24-48 h de la ingesta.

maculopapular.

Urticaria no inmediata.

Erupción fija por droga.

NET/SSJ.

DRESS.

PEGA.

Reacción específica de un órgano.
Alta frecuencia.

Eritema difuso y pápulas que pueden evolucionar a vesículas; a veces, acompañadas de angioedema.

Habones urticarianos típicos sin angioedema.

Los AINE se encuentran entre sus causas más frecuentes.

Parches eritematosos que se repiten, principalmente, en el mismo sitio en la reexposición al medicamento causante.

Ibuprofeno, naproxeno, dipirona.

Destrucción generalizada de la piel y mucosas. AAS-paracetamol.

Fiebre, rash e hipereosinofilia acompañados de hepatitis e insuficiencia renal.

Ibuprofeno e inhibidores selectivos de la COX-2.

Micropústulas estériles no foliculares, sobre un fondo eritematoedematoso. Paracetamol-bufexamac.

Hepatitis, neumonitis o meningitis asépticas.

SNIUAA: urticaria o angioedema y / o anafilaxia inducida por un solo antiinflamatorio no esteroideo (del inglés selective nonsteroidal antiinflammatory drugs induced urticaria/angioedema or anaphylaxis); IgE: inmunoglogulina E; AINE: antiinflamatorio no esteroideo; COX-1: ciclooxigenasa-1; SNIDR: reacción selectiva retardada inducida por antiinflamatorio no esteroideo (del inglés selective non-steroidal antiinflammatory drugs induced delayed reactions); LT: linfocito T; NET/SSJ: necrólisis epidérmica tóxica / síndrome de Stevens-Johnson; AAS: ácido acetil salicílico; DRESS: reacción a drogas con eosinofilia y síntomas sistémicos (del inglés drug rash with eosinophilia and systemic symptoms); COX-2: ciclooxigenasa-2; PEGA: pustulosis exantemática generalizada aguda. 


\section{Laboratorio}

Se dispone de un número limitado de pruebas in vitro, tales como test de activación de basófilos (basophil activation test; BAT, por sus siglas en inglés), prueba de estimulación de antígeno celular (cellular antigen stimulation test; CAST, por sus siglas en inglés), test de transformación linfocitaria, enzyme-linked immunospot assay (ELISpot); sin embargo, su sensibilidad, especificidad y valores predictivos positivos y negativos no están bien establecidos en los niños. ${ }^{23,27}$

Si se sospecha anafilaxia, para su confirmación, se puede medir la triptasa sérica. Debe realizarse dentro de las 2-5 horas del inicio de la reacción y ser repetida a los 2-7 días para conocer la cifra basal del niño. ${ }^{28-30}$

\section{Pruebas cutáneas}

La prueba cuánea de lectura inmediata (PCLI) o prick test es útil ante síntomas como urticaria, angioedema y / o anafilaxia inducidos por un solo AINE (selective non-steroidal antiinflammatory drugs induced urticarialangioedema or anaphylaxis; SNIUAA, por sus siglas en inglés), en los que se sospecha un mecanismo mediado por IgE. Se ha utilizado para el diagnóstico de reacciones inmediatas a dipirona y paracetamol en los niños. La prueba intradérmica puede ser más sensible. Una prueba cutánea negativa no excluye el diagnóstico. Para confirmar la negatividad, se debe realizar una provocación oral. Las formulaciones de medicamentos y la concentración para las pruebas cutáneas en los niños deben validarse más. ${ }^{23,27}$

En las reacciones retardadas, se pueden considerar las pruebas intradérmicas con lectura tardía. Aunque las pruebas de parches con AINE específicos no han sido suficientemente estandarizadas, pueden ser útiles para reacciones tardías, en particular, en casos de dermatitis de contacto y eritema fijo por drogas. ${ }^{13}$

\section{Prueba de provocación controlada}

Para confirmar el diagnóstico de hipersensibilidad a AINE en los niños, la prueba de provocación controlada (PPC) sigue siendo el estándar de oro. En la mayoría de los casos, un procedimiento abierto o simple ciego es suficiente para establecerlo. ${ }^{23,31,32}$

Se puede realizar para confirmar la hipersensibilidad a un determinado AINE, con otros AINE que no sean causales (generalmente, prueba de desafío con aspirina) para confirmar o excluir la reactividad cruzada y con el fármaco alternativo con más probabilidad de ser tolerado. Debe ser realizada por un especialista experimentado. ${ }^{13}$

TABLA 4. Manifestaciones clinicas en las reacciones de intolerancia cruzadas ${ }^{23,25}$

- Mecanismo no inmunológico desencadenado por la combinación entre la inhibición de la COX-1 y un desequilibrio en el metabolismo del ácido araquidónico.

- Inmediatas de minutos a varias horas pos ingesta del AINE.

Entidades clínicas

NERD

NECD

NIUA

Puntos claves

Es la segunda causa en frecuencia de alergia a AINE en pediatría. Broncoespasmo, disnea y/u obstrucción nasal o rinorrea.

La enfermedad respiratoria subyacente más frecuente es la rinosinusitis. La tríada ASA no se expresa completamente en niños y adolescentes, sino que involucra las vías aéreas superiores/inferiores en diversos grados. Más frecuente en pacientes atópicos, con prick test positivo a ácaros.

Urticaria y/o angioedema en pacientes con UCE.

Es rara en los niños.

Se da, particularmente, con los inhibidores potentes de la COX-1.

Entidad más frecuente de alergia a AINE en pediatría.

Especialmente, en niños atópicos.

Se caracteriza por angioedema facial seguido de urticaria generalizada, aunque pueden aparecer simultáneamente.

Puede asociarse a síntomas respiratorios, sin caída de la presión arterial, simulando una anafilaxia, pero su mecanismo no es mediado por IgE.

NERD: enfermedad respiratoria exacerbada por antiinflamatorios no esteroideos (del inglés non-steroidal antiinflammatory drugs exacerbated respiratory disease); AINE: antiinflamatorio no esteroideo; ASA: asma, rinitis/poliposis nasal e intolerancia a la aspirina; NECD: enfermedad cutánea exacerbada por antiinflamatorios no esteroideos (del inglés non-steroidal antiinflammatory drugs exacerbated cutaneous disease); UCE: urticaria crónica espontánea; COX-1: ciclooxigenasa-1; NIUA: urticaria o angioedema inducidos por antiinflamatorios no esteroideos (del inglés non-steroidal antiinflammatory drugs induced urticaria/angioedema). 
La PPC es útil para identificar una droga alternativa tolerada y / o el AINE responsable de una reacción alérgica. Tiene un valor predictivo negativo muy alto (del 97,8\%), por lo que su negatividad permite el uso seguro de la droga involucrada. El valor predictivo positivo es cercano al $100 \% .{ }^{13}$ Está contraindicada en los pacientes que presentaron anafilaxia, necrólisis epidérmica tóxica o síndrome de Stevens-Johnson y en los asmáticos con flujo espiratorio forzado en el primer segundo (forced expiratory volume in one second; FEV1, por sus siglas en inglés) $<1,5$ litros.
Algunas drogas, entre las que se destacan antihistamínicos, $\beta 2$ agonistas inhalados, anticolinérgicos, corticoides y antileucotrienos, pueden enmascarar la reacción, por lo que deben ser suspendidas antes de realizar la PPC. ${ }^{33}$ Se requerirán pruebas de la función pulmonar previas a la administración de cada nueva dosis. Deben ser efectuadas por equipos experimentados en un entorno apropiado que garantice la seguridad de los pacientes. ${ }^{13,23,34,35}$

En la Tabla 5, se presentan las ventajas y desventajas de cada método diagnóstico.

TABLA 5. Métodos diagnósticos: ventajas y desventajas ${ }^{13,23,24}$

\begin{tabular}{|c|c|c|}
\hline Método diagnóstico & Ventajas & Desventajas \\
\hline IgE sérica específica & Útil para reacciones a un solo AINE. & $\begin{array}{l}\text { - Panel limitado de medicamentos. } \\
\text { • No está validada. }\end{array}$ \\
\hline BAT & $\begin{array}{l}\text { - Sensibilidad del } 60 \% \text {-VPP del } 100 \% \text {. } \\
\text { • Elevada reproducibilidad. }\end{array}$ & - No accesible. \\
\hline CAST & - Sensibilidad del $38 \%$. & - No validada. \\
\hline PCLI o prick test & $\begin{array}{l}\text { • Técnica sencilla, segura. } \\
\text { - Sensibilidad moderada baja para reacciones } \\
\text { de tipo inmediato a los medicamentos. }\end{array}$ & $\begin{array}{l}\text { - No existe antígeno adecuado. } \\
\text { - Interpretación del resultado: requiere } \\
\text { dilución acorde para no inducir una } \\
\text { reacción irritativa (falsos positivos). } \\
\bullet \text { No estandarizada. } \\
\text { - Su negatividad no excluye el diagnóstico. }\end{array}$ \\
\hline TPA & Útil en reacciones retardadas. & $\begin{array}{l}\text { - Falsos negativos por técnica irregular o porque } \\
\text { la reacción fue por metabolitos del fármaco. } \\
\text { - La lectura debe realizarse en } 2 \text { tiempos. }\end{array}$ \\
\hline PPC & $\begin{array}{l}\text { • Confirma el diagnóstico. } \\
\text { • Descarta alergia al fármaco. } \\
\text { - Evita la exclusión de AINE por largos períodos. } \\
\text { - Permite la búsqueda alternativa farmacológica } \\
\text { en asmáticos con intolerancia a AAS. }\end{array}$ & $\begin{array}{c}\text { • Requiere que el paciente se } \\
\text { encuentre clínicamente estable al momento } \\
\text { de su realización. } \\
\text { • No puede realizarse durante el embarazo } \\
\text { o con comorbilidades (enfermedad cardíaca, } \\
\text { hepática o renal grave). } \\
\text { - Necesita personal médico entrenado para efectuar } \\
\text { el protocolo en la unidad hospitalaria. } \\
\text { • Puede reproducir reacciones graves. } \\
\text { • En los menores, requiere el } \\
\text { consentimiento de los padres. } \\
\text { - No puede realizarse en pacientes con } \\
\text { anafilaxia previa. }\end{array}$ \\
\hline
\end{tabular}

IgE: inmunoglobulina E; BAT: prueba de activación de basófilos; CAST: determinación de sulfidoleucotrienos específica de antígeno; PCLI: prueba cutánea de lectura inmediata; TPA: test de parche atópico; PPC: prueba de provocación controlada; AINE: antiinflamatorio no esteroideo; VPP: valor predictivo positivo; AAS: ácido acetil salicílico.

TABLA 6. Clasificación de los antiinflamatorios no esteroideos según el grupo químico ${ }^{34}$

\begin{tabular}{ll}
\hline Grupo químico & AINE \\
\hline Ácidos salicílicos & Ácido acetil salicílico, ácido salicílico, diflunisal \\
Ácidos acéticos & Indometacina, sulindac, ketorolac, etodolac, tolmetina \\
Ácidos propiónicos & Ibuprofeno, naproxeno, ketoprofeno, dexketoprofeno, fenoprofeno, fenbufeno, \\
& flurbiprofeno, benoxaprofeno, suprofeno, pirprofeno, indoprofeno \\
Fenilacéticos & Diclofenac, aceclofenac \\
Ácidos enólicos & Meloxicam, piroxicam, tenoxicam \\
Ácidos fenámicos & Ácido mefenámico, ácido flufenámico \\
Acetaminofenol & Paracetamol \\
Derivado de sulfonanilida & Nimesulida \\
Pirazolonas & Dipirona, fenilbutazona, propifenazona \\
Coxibs & Celecoxib, etoricoxib \\
\hline
\end{tabular}




\section{TRATAMIENTO}

Deberá evitarse la droga o las drogas involucradas y proporcionar alternativas seguras para cada paciente en particular. Se brindará información escrita y oral para facilitar al pediatra y a la familia los futuros tratamientos. ${ }^{23}$

En los pacientes con reacciones alérgicas o selectivas a un fármaco, se evitará el fármaco responsable y aquellos relacionados químicamente. Estos pacientes pueden tolerar otros AINE no relacionados químicamente (Tabla 6), con independencia de su capacidad para inhibir la COX-1. ${ }^{36}$ En los pacientes con intolerancia cruzada, deberán tomarse en cuenta los diferentes grados de selectividad en relación con la inhibición de la COX-1 y la COX-2 (Tabla 2).

Para el tratamiento de los cuadros febriles, la mayoría de los niños con intolerancia cruzada a AINE pueden tolerar bajas dosis de paracetamol. ${ }^{37}$ En aquellos que reaccionan al paracetamol, los medios físicos y una buena hidratación con bebidas frías pueden tener una eficacia parcial. ${ }^{23} \mathrm{Si}$ se busca un efecto antiinflamatorio, los corticoides son la alternativa más importante. ${ }^{23}$
Algunos inhibidores preferenciales de la COX2 parecen ser seguros en el 80-85\% de los niños con intolerancia a AINE. ${ }^{38-41}$ Existen reportes de su uso en menores de 12 años con enfermedades reumatológicas. ${ }^{42}$ Tienen acción antifebril y antiinflamatoria. Sin embargo, estos fármacos no han sido aprobados para menores de 12 años de edad y no se cuenta con formulaciones pediátricas.

Los derivados opioides pueden ser una alternativa analgésica, sobre todo, en los pacientes posquirúrgicos. También, los inhibidores de la COX-2 ${ }^{43-44}$ con las salvedades ya mencionadas. Con respecto al meloxicam, ha demostrado ser seguro en pacientes adultos con intolerancia a AINE; sin embargo, no debería utilizarse en los menores de 15 años de edad. ${ }^{45}$

Cuando la administración de un AINE es imprescindible, como en el caso de pacientes que requieren antiagregación o tratamiento antiinflamatorio crónico con AINE (enfermedades coronarias, como el Kawasaki, y/o enfermedades reumatoides), se puede realizar un proceso de desensibilización ${ }^{46}$ (Tabla 7).

TABLA 7. Criterios para la desensibilización en reacción a antiinflamatorios no esteroideos ${ }^{46}$

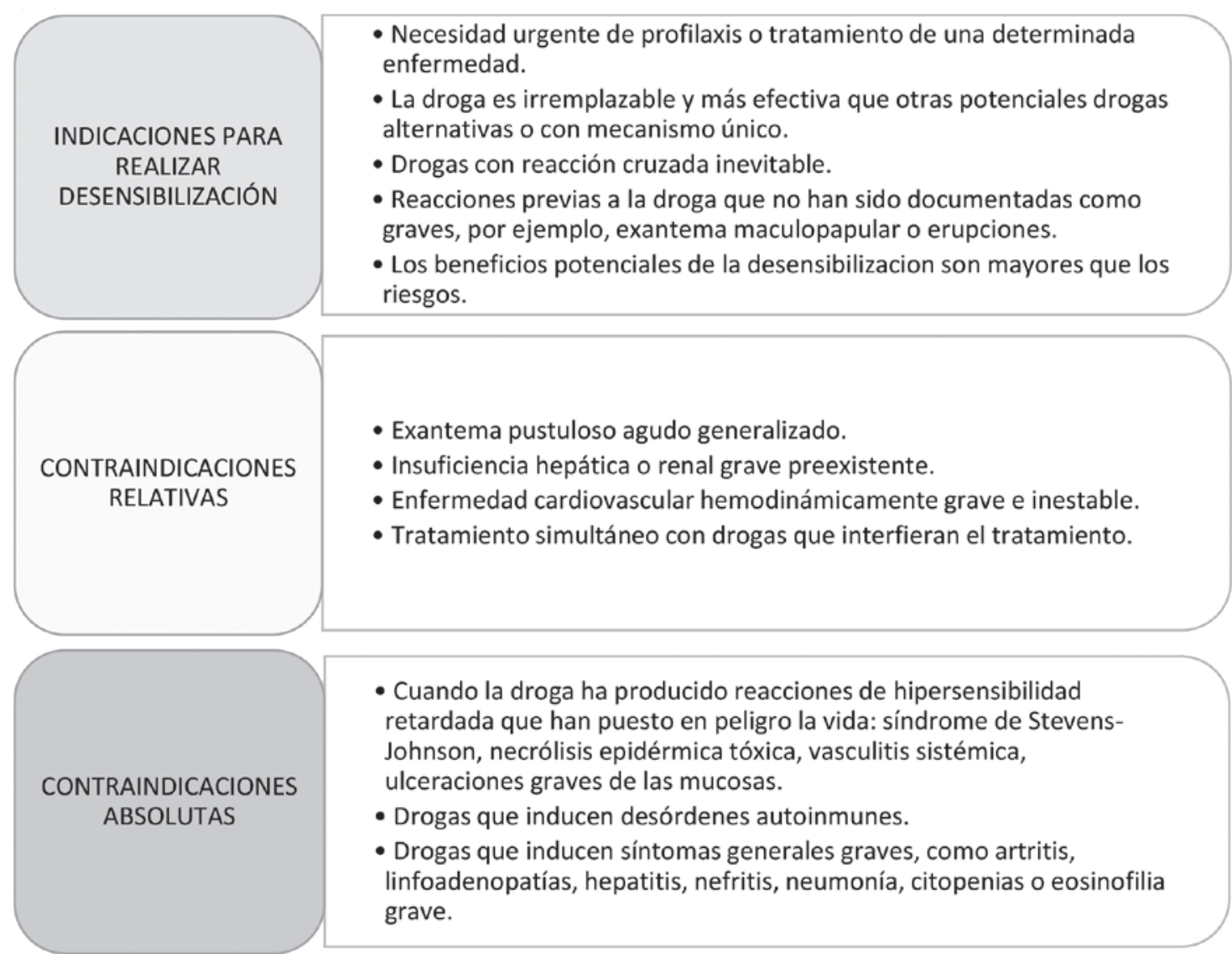


La enfermedad respiratoria exacerbada por AINE es una indicación posible, aunque poco frecuente, de desensibilización en los niños. ${ }^{23}$ Debido a la escasa evidencia de desensibilización con AINE en los niños, los protocolos se adaptan de aquellos diseñados para adultos. ${ }^{13,47}$

\section{PREVENCIÓN}

Luego de la confirmación diagnóstica, es importante realizar un detallado informe escrito para prevenir futuras reacciones. Estas indicaciones deben ser comprendidas por el paciente y sus cuidadores, y ser difundidas en el ambiente familiar, escolar, social y lúdico. Se deberá identificar específicamente la droga involucrada, las marcas comerciales que la contienen y si existen reacciones cruzadas con alimentos a fines de evitar la ingesta de ellos. ${ }^{48}$ Debe especificar los síntomas que provoca la reacción, la indicación de la medicación inicial en caso de anafilaxia y el contacto telefónico de acceso a la atención médica específica. Se propone que el paciente lleve consigo una historia clínica que consigne toda esa información. Internacionalmente, se la ha denominado pasaporte de la alergia a fármacos. ${ }^{49}$

\section{CONCLUSIONES}

Debido a la amplia utilización de AINE en pediatría, la sospecha de alergia a estas drogas es algo cotidiano para el pediatra. La confusión con síntomas propios de los procesos virales y la variabilidad de la clínica hacen del diagnóstico de certeza de esta patología un verdadero desafío. La confirmación es fundamental para no etiquetar como alérgico a un niño que no lo es y para no exponer a riesgos innecesarios a quien está sensibilizado.

Es imprescindible definir estrategias para cada paciente en particular, que surgirán de una correcta evaluación alergológica. Si bien queda mucho por avanzar en el estudio de esta patología, el especialista en Alergia e Inmunología Infantil cuenta con las herramientas necesarias para arribar a un diagnóstico de certeza que mejorará la atención y la calidad de vida de los niños.

\section{Filiación de los autores}

Dra. María P. Sarraquigne: Facultad de Ciencias Médicas, Universidad Nacional de Rosario Sanatorio de Niños. Rosario, Santa Fe, Argentina. Dra. Andrea I. Mariño: Hospital Municipal de Agudos Dr. Leónidas Lucero- Carrera de
Medicina de la Universidad Nacional del Sur. Bahía Blanca, Buenos Aires, Argentina.

Dr. Ricardo Saranz: Clínica Universitaria Reina Fabiola, Universidad Católica de Córdoba (UCC) - Catedra de Inmunología, Facultad de Ciencias de la Salud, UCC. Córdoba, Córdoba, Argentina. Dr. Mauricio Colella: Hospital Escuela Eva Perón, Granadero Baigorria - Facultad de Ciencias Médicas, Universidad Nacional de RosarioSanatorio de Niños. Rosario, Santa Fe, Argentina. Dra. Karina López: Hospital de Niños Víctor J. Vilela - Hospital Alverdi - Estación Infancia. Rosario, Santa Fe, Argentina.

Dra. María del P. Bovina Martijena: Sanatorio Allende. Córdoba, Córdoba, Argentina.

Dr. Claudio Parisi: Hospital Italiano de Buenos Aires. Ciudad Autónoma de Buenos Aires, Argentina.

Dr. Julio Orellana: Hospital de Niños de la Santísima Trinidad - Sanatorio Allende. Córdoba, Córdoba, Argentina.

Dr. Claudio Agüero: Instituto Dr. Ricardini Policlínico Grupo Oroño. San Nicolás, Buenos Aires, Argentina.

Dra. Viviana Seisdedos: Hospital Municipal de Agudos Dr. Leónidas Lucero. Bahía Blanca, Buenos Aires, Argentina.

Dr. Alejandro Lozano: Clínica Universitaria Reina Fabiola, Universidad Católica de Córdoba (UCC) - Catedra de Inmunología, Facultad de Ciencias de la Salud, UCC. Córdoba, Córdoba, Argentina. Dr. Jorge F. Máspero: Fundación Cidea - Hospital Alemán. Ciudad Autónoma de Buenos Aires, Argentina.

Dr. Raúl V. Boudet: Centro de Alergia e Inmunología Infantil. Río Cuarto, Córdoba, Argentina.

Dr. Juan M. Suárez García: Hospital de Niños Debilio Blanco Villegas. Tandil, Buenos Aires, Argentina.

Dra. Gloria Bandin: Centro de Especialidades Médicas Pediátricas - Consultorios de la Pueyrredón. Rosario, Santa Fe, Argentina.

Dra. Marcela García: Hospital de Niños Sor María Ludovica de La Plata, Buenos Aires, Argentina.

Dra. María E. Gervasoni: Hospital de Niños Víctor J. Vilela - Centro Respiratorio Infantil. Rosario, Santa Fe, Argentina.

Dra. Natalia Luconi: Instituto Medicina Infantil. Luján de Cuyo, Mendoza, Argentina.

Dra. Mónica Matta Rufolo: Hospital General de Agudos Donación Francisco Santojanni, Ciudad Autónoma de Buenos Aires, Argentina.

Dr. Víctor Skrie: Hospital de Niños de la Santísima 
Trinidad. Córdoba, Córdoba, Argentina. Dr. Martín Bózzola: Hospital Británico Buenos Aires. Ciudad Autónoma de Buenos Aires, Argentina.

Dr. Norberto Procopio: Centro Respiratorio Infantil. Hospital Español. Rosario, Santa Fe, Argentina. Dr. Antonio Sánchez Segovia: Hospital San Martín, Paraná, Entre Ríos, Argentina.

Dra. Irene Araoz: Sanatorio Mayo, Santa Fe, Santa $\mathrm{Fe}$, Argentina.

Dra. María C. Cassaniti: Consultorio privado. Paraná, Entre Ríos, Argentina.

Dra. Elsa Mindel: Sociedad Argentina de Pediatría filial Rosario. Rosario, Santa Fe, Argentina.

\section{REFERENCIAS}

1. Cortada-Macías J, López-Serrano M, Blasco-Sarramián A, Mayorga C, et al. Introducción, conceptos generales, epidemiología. Fisiopatología: los fármacos como antígenos. In: Peláez-Hernández A, Dávila-González I. Tratado de Alergología. Madrid: Ergón; 2007.Págs.1297-324.

2. Vervloet D, Durham S. Adverse reactions to drugs. BMJ. 1998; 316(7143):1511-4.

3. Gell P, Coombs R. Clinical aspects of immunology. Oxford: Blackwell Science; 1964.

4. Guvenir H, Dibek-Misirlioglu E, Vezir E, Toyran M, et al. Nonsteroidal anti-inflamatory drug hypersensitivity among children. Allergy Asthma Proc. 2015; 36(5):386-93.

5. Cardona R, Ramírez R, Reina Z, Escobar M, et al. Alergia e intolerancia a antiinflamatorios no esteroides: desensibilización exitosa en tres casos y revisión de la literatura. Biomédica. 2009; 29(2):181-90.

6. CaubetJC, Eigenmann P. Diagnosticissues in pediatric drug allergy. Curr Opin Allergy Clin Immunol. 2012; 12(4):341-7.

7. Atanaskovic-Markovic M, Caubet J. Management of drug hypersensitivity in the pediatric population. Expert Rev Clin Pharmacol. 2016; 9(10):1341-9.

8. Kowalski M, Makowska J, Blanca M, Bavbek S, et al. Hypersensitivity to nonsteroidal anti-ininflammatory drugs(NSAIDs)-classification, diagnosis and management: review of the EAACI/ENDA(\#) and GA2LEN/HANNA*. Allergy. 2011; 66(7):818-29.

9. Blanca-López N, Pérez-Alzate D, Andreu I, Doña I, et al. Immediate hypersensitivity reactions to ibuprofen and other arylpropionic acid derivates. Allergy. 2016;71(7):104656.

10. Atanasković-Marković M, Gaeta F, Gavrović-Jankulović M, Cirkovic Velickovic T, et al. Diagnosing multiple drug hypersensitivity in children. Pediatr Allergy Immunol. 2012; 23(8):785-91.

11. Toche Pinaud P. Alergia a antiinflamatorios noesteroidales. Medwave. 2010; 10(9):e4774.

12. Porto Arceo J. Particularidades de la intolerancia AINEs en niños. Allergol Immunopathol (Madr). 2003; 31(3):109-25.

13. Kowalski M, Asero R, Bavbek S, Blanca M, et al. Classification and practical approach to the diagnosis and management of hypersensitivity to nonsteroideal antiinflammatory drugs. Allergy. 2013; 68(10):1219-32.

14. Topal E, Celiksoy M, Catal F, Gamze Sayan Y, et al. The value of the clinical history for the diagnosis of immediate nonsteroidal anti-inflammatory drug hypersensitivity and safe alternative drugs in children. Allergy Asthma Proc. 2016; 37(1):57-63.

15. Blanca-López N, Cornejo-García J, Pérez-Alzate D,
Pérez-Sánchez N, et-al. Hypersensitivity Reactions to Nonsteroidal Anti-inflammatory Drugs in Children and Adolescents: Selective Reactions. J Investig Allergol Clin Immunol. 2015; 25(6):385-95.

16. Modena B, White A, Woessner K. Aspirin and nonsteroidal antiinflamatory drugs hypersensitivity and management. Immunol Allergy Clin North Am. 2017; 37(4):727-49.

17. White A, Stevenson D. Aspirin-exacerbated respiratory disease. N Engl J Med. 2018; 379(11):1060-70.

18. Cahill K, Boyce J. Aspirin-exacerbated respiratory disease: Mediators and mechanisms of a clinical disease. J Allergy Clin Immunol. 2017; 139(3):764-6.

19. Liu T, Kanaoka Y, Barret N, Feng C, et al. Aspirinexacerbated respiratory disease involves a cysteinyl leukotriene-driven IL-33-mediated mast cell activation pathway. J Immunol. 2015; 195(8):3537-45.

20. Swierkosz T, Jordan L, McBride M, McGough K, et al. Actions of paracetamol on cyclooxygenases in tissue and cell homogenates of mouse and rabbit. Med Sci Monit. 2002; 8(12):BR496-503.

21. Botting R. Mechanism of action of acetaminophen: is there a cyclooxygenase 3? Clin Infect Dis. 2000;31(Suppl 5):S20210 .

22. Sánchez-Borges M, Kidon M. Reactions to nonsteroidal anti-inflammatory drugs in children. J Allergy Clin Immunol Pract. 2018; 6(4):1236-7.

23. Kidon M, Blanca-López N, Gomes E, Terreehorst I, et al. EAACI/ENDA Position Paper:Diagnosis and management of hypersensitivity reactions to non-steroidal antiinflammatory drugs (NSAIDs) in children and adolescents. Pediatr Allergy Immunol. 2018; 29(5):469-80.

24. García-Aviles M, Sanz-Larruga M, Lobera-Labairu T, Gracia-Bara M, et al. Diagnóstico de Alergia a Fármacos. In: Pelaez Hernandez A, Dávila González I. Tratado de Alergología. Madrid: Ergon; 2007.Págs.1349-68.

25. Blanca-López N, Cornejo-García J, Plaza-Serón M, Dona I, et al. Hypersensitivity to Nonsteroidal Anti-inflammatory Drugs in Children and Adolescents: Cross-Intolerance Reactions. J Investig Allergol Clin Immunol. 2015; 25(4):25969.

26. Heelan K, Shear N. Cutaneous Drug Reactions in Children: an update. Paediatr Drugs. 2013; 15(6):493-503.

27. Torres M, Barrionuevo E, Kowalski M, Blanca M. Hypersensitivity Reactions to Nonsteroidal AntiInflammatory Drugs. Immunol Allergy Clin N Am. 2014; 34(3):507-24.

28. Brockow K, Przybilla B, AbererW, Bircher A, et al. Guideline for the diagnosis of drug hypersensitivity reactions: S2KGuideline of the GermanSociety for Allergology and Clinical Immunology (DGAKI) and the German Dermatological Society (DDG) in collaboration with the Association of German Allergologists (AeDA), the German Society for Pediatric Allergology and Environmental Medicine(GPA), the German Contact Dermatitis Research Group (DKG), the Swiss Society for Allergy and Immunology (SGAI), the Austrian Society for Allergology and Immunology (ÖGAI), the German Academy of Allergology and Environmental Medicine (DAAU), the German Center for Documentation of Severe Skin Reactions and the German Federal Institute for Drugs and Medical Products (BfArM). Allergo J Int. 2015; 24(3):94-105.

29. Echeverría Zudaire L, Del Olmo de la Lama M, Santana Rodríguez C. Anafilaxia en Pediatría. Protoc Diagn Ter Pediatr. 2013; 1:63-80.

30. De Schryver S, Halbrich M, Clarke A, La Vieille S, et al. Tryptase levels in children presenting with anaphylaxis: Temporal trends and associated factors. I Allergy Clin Immunol. 2015; 137(4):1138-42. 
31. Gomes E, Brockow K, Kuyucu S, Saretta F, et al. Drug hypersensitivity in children: report from the pediatric task force of the EAACI Drug Allergy Interest Group. Allergy. 2016; 71(2):149-61.

32. Alves C, Romeira A, Abreu C, Carreiro-Martins C, et al. Non-steroidal anti-inflammatory drug hypersensitivity in children. Allergol Immunopathol (Madr). 2017; 45(1):40-7.

33. Bousquet PJ, Gaeta F, Bousquet-Trouanet L, Lefrant JY, et al. Provocation Tests in Diagnosing Drug Hypersensitivity. Curr Pharm Des. 2008; 14(27):2792- 802.

34. Ortega-Rodríguez N, Quiralte-Enriquez J, Frag-Lázaro J, Palacios-Colom L. Reacciones adversas a los AINE: alergia e intolerancia. In: Peláez Hernández A, Dávila González I. Tratado de Alergología. Madrid: Ergon; 2007.Págs.1461-82.

35. Nizankowska-Mogilnicka E, Bochenek G, Mastalerz L, Swierczynska M, etal.EAACI/GA2LEN guideline: aspirin provocation tests for diagnosis of aspirin hypersensitivity. Allergy. 2007; 62(10):1111-8.

36. Blanca López N, Pérez AlzateD, CantoG, Blanca M. Practical approach to the treatment of NSAID hypersensitivity. Expert Rev Clin Immunol 2017; 13(11): 1017-27.

37. Kidon M, Liew W, Chiang W, Lim S, et al. Hypersensitivity to paracetamol in Asian children with early onset of nonsteroidal anti-inflammatory drug allergy. Int Arch Allergy Immunol. 2007; 144(1):51-6.

38. CorzoJ,ZamboninoM, MuñozC,Mayorga C, etal.Tolerance to COX-2 inhibitors in children with hypersensitivity to nonsteroidal anti-inflammatory drugs. Br J Dermatol. 2014; 170(3):725-9.

39. Sánchez Borges M, Capriles-Hulett A, Caballero-Fonseca F, Pérez C. Tolerability to new COX-2 inhibitors in NSAIDsensitive patients with cutaneous reactions. Ann Allergy Asthma Immunol. 2001; 87(3):201-4.

40. Sánchez-Borges M, Caballero-Fonseca F, Capriles-HulettA. Tolerance of nonsteroidal anti-inflammatory drug-sensitive patients to the highly specific cyclooxygenase 2 inhibitors rofecoxib and valdecoxib. Ann Allergy Asthma Immunol. 2005; 94(1):34-8.

41. Loh W, Lim H, Rao R, Goh A, et al. Tolerance to etoricoxib in children with nonsteroidal anti-inflammatory drug hypersensitivity. Asia Pac Allergy. 2015; 5(1):40-6.

42. Levy D, Imundo L. Nonsteroidal Anti-Inflammatory Drugs: A survey of practices and concerns of pediatric medical and surgical specialists and a summary of available safety data. Pediatr Rheumatol Online J. 2010; 8:7.

43. Murto K, Lamontagne C, McFaul C, MacCormick $\mathrm{J}$, et al. Celecoxib pharmacogenetics and pediatric adenotonsillectomy: a double-blinded randomized controlled study. Can J Anaesth. 2015; 62(7):785-97.

44. Li X, Zhou M, Xia Q, Li J. Parecoxib sodium reduces the need for opioids after tonsillectomy in children: a doubleblind placebo-controlled randomized clinical trial. Can J Anaesth. 2016; 63(3):268-74.

45. Vázquez-Cortés S, Vázquez-Fuertes L, Rodríguez-Alvarez M, Reig Rincón de Arellano I, et al. Tolerancia a celecoxib y meloxicam en pacientes con intolerancia a analgésicos no esteroideos. An Med Interna. 2008; 25(4):163-7.

46. SchererK, Brockow K, AbererW, GooiJ, etal.Desensitization in delayed drug hypersensitivity reactions - an EAACI position paper of the Drug Allergy Interest Group. Allergy. 2013; 68(7):844-52.

47. Cernadas JR, Brockow K, Romano AW, Aberer W, et al. General considerations on rapid desensitization for drug hypersensitivity - a consensus statement. Allergy. 2010; 65(11):1357-66.

48. Barbaud A. Place of excipients in Systemic drug allergy. Immunol Allergy Clin North Am. 2014; 34(3):671-9.

49. Brockow K, Aberer W, Atanaskovic-Markovic M, Bavbek $S$, et al. Drug allergy passport and other documentation for patients with drug hypersensitivity - An ENDA/EAACI Drug Allergy Interest Group Position Paper. Allergy. 2016; 71(11):1533-9. 